\title{
Wear of the PG-CP4 powder coating applied to HVG steel and subjected to laser treatment
}

\author{
Vladimir Gusev ${ }^{1, *}$, Valentin Morozov ${ }^{1}$, Alexey Morozov ${ }^{1}$, and Dmitry Gavrilov ${ }^{1}$ \\ ${ }^{1}$ Vladimir State University named after Alexander and Nikolay Stoletovs, Russian Federation
}

\begin{abstract}
The article considers the influence of the radiation power $W$, the longitudinal feed Spr of the laser beam and the distance $L$ from the protective glass of the laser focusing head to the workpiece on the $P G-C P 4$ powder coating absolute wear which was applied to $H V G$ steel by plasma and subjected to laser treatment. A multi-factor model is established that relates the absolute wear of $P G-C P 4$ coating to the input process factors and allows you to assign a laser treatment mode to provide the minimum wear of the processed surface layer. The greatest influence on coating absolute wear is provided by the longitudinal feed of laser beam, with which the wear increases. Compared to Spr, the influence of factor $W$ on the coating absolute wear is $24 \%$ less, and the factor $L$ influence is 4.4 times less. The minimum absolute wear of the coating occurs at $W=5 \mathrm{~kW}$, $S p r=40 \mathrm{~mm} / \mathrm{s}, L=85 \mathrm{~mm}$. The results of the research are recommended for use in enterprises that implement the processes of plasma and laser processing of materials, as well as in design organizations that develop modern technological laser systems.
\end{abstract}

\section{Introduction}

$H V G$ steel is characterized by low deformation during quenching, significant strength and moderate cost, so it is widely used for the manufacture of cutting tools, dies, punches etc. High values of wear resistance under abrasive wear are obtained [1], the influence of the laser treatment process on the microstructure and the formation of a thermal influence zone is investigated [2]. It is noted in [3] that the classical technologies of local thermal hardening of steels and alloys: light-beam, plasma, laser and others allow to increase the service life of the cutting tool. These methods have now almost exhausted their capabilities to increase the efficiency of hardening and do not allow to radically increase wear resistance. The development of hybrid heat treatment technologies (laser-plasma, etc.) is more promising than the classical methods of local thermal hardening.

Combined laser-plasma treatment of metals has been studied [4], the wear resistance of die steels after volumetric and plasma quenching [5-8], the heat treatment regime for heavily loaded cold deformation dies has been justified [9]. Using the scientific provisions [10], multifactor models of hardness, friction coefficient and wear for steel $40 \mathrm{H} 13$ were obtained $[11,12]$.

\footnotetext{
*Corresponding author: prof_gusev@mail.ru
} 
The fields of surface structuring application by lasers [13], the effect of laser quenching and heat treatment on the wear resistance of cemented steel 20HN3A [14], laser modification of tribological properties of steels [15-17] and coatings [18] are considered. An increase in the abrasive wear resistance of steels is achieved by using multi-channel laser hardening [19]. The combined treatment of $\mathrm{NiCrBSi}$ coating leads to the formation of large carbides and chromium borides, which increases their wear resistance [20]. The considered works allow to increase the efficiency of laser and plasma processing of metals and alloys, the proposed models are mostly single-factor ones that do not reflect the course of multifactorial processes.

The aim of the study is to develop a multi-factor model of absolute wear of the $P G-C P 4$ powder coating subjected to plasma spraying on $H V G$ steel and further laser treatment.

To achieve this goal, the choice of independent factors of the HVG steel laser processing, which have a dominant influence on the optimization parameter (absolute wear of the coating), is justified. As a result of statistical processing of experimental data, a multifactorial model was established that relates the absolute wear of the PG-CP4 coating to the radiation power, the longitudinal feed of the laser beam and the distance from the protective glass of the laser head to the treated surface of the sample.

\section{Materials and Methods}

To apply PG-CP4 powder to samples made of $H V G$ steel, an $U P N-350$ plasma installation was used. The powder includes (13.5-16.5) \% chromium, (2.5-3.5) \% silicon, (2.0-2.8) \% boron, up to $5 \%$ iron, nickel is the basis of the powder. The samples were treated on the SVAROG-1-5D laser complex (Russia) on the modes shown in Table 1 . The radiation power $W$, the longitudinal feed Spr of the laser beam, and the distance $L$ from the protective glass of the laser head to the treated surface of the sample were selected as independent input factors of the laser processing. As an optimization parameter, the absolute wear of the protective coating was selected. Was implemented a plan for experiment $N=2^{\wedge} k=2^{\wedge} 3=8$, where $k$ is the factors number.

\section{Results}

The values of the coating $U$ absolute wear are presented in the rightmost column of Table 1 . Depending on the laser treatment mode, reflow $(+)$ of the surface layer or its absence (-) was observed.

Table 1. A coating wear of steel $H V G$ as a function of laser treatment modes

\begin{tabular}{|c|c|c|c|c|}
\hline \multirow{2}{*}{ Samples } & \multicolumn{3}{|c|}{ Independent factors } & Parameter \\
\cline { 2 - 5 } & $\begin{array}{c}\text { Power } \\
W, \mathrm{~kW}\end{array}$ & $\begin{array}{c}\text { Feed } S p r, \\
\mathrm{~mm} / \mathrm{s}\end{array}$ & $\begin{array}{c}\text { Distance } L, \\
\mathrm{~mm}\end{array}$ & $\begin{array}{c}\text { Wear, } \\
\mu \mathrm{m}\end{array}$ \\
\cline { 2 - 5 } & $x_{1}$ & $x_{2}$ & $x_{3}$ & $U$ \\
\hline $3.7(+)$ & 5 & 25 & 85 & 40 \\
\hline $3.1(-)$ & 2 & 25 & 85 & 74 \\
\hline $3.2(-)$ & 5 & 10 & 85 & 43 \\
\hline $3.6(-)$ & 2 & 10 & 85 & 44 \\
\hline $3.3(-)$ & 5 & 25 & 60 & 64 \\
\hline $3.4(-)$ & 2 & 25 & 60 & 52 \\
\hline $3.8(+)$ & 5 & 10 & 60 & 56 \\
\hline $3.5(-)$ & 2 & 10 & 60 & 50 \\
\hline \multicolumn{5}{|l}{ Wear of $H V G$ steel in the delivery state } \\
\hline
\end{tabular}


The effect of the radiation power $W$ and the longitudinal feed Spr of the laser beam on the absolute wear of the coating applied by plasma to the $H V G$ chrome steel and subjected to laser treatment is characterized by a multifactorial dependence of the form:

$$
U=49.33-2.67 * W+0.7 * \operatorname{Spr}
$$

Equation (1) can be graphically interpreted by a 3D-XYZ surface-graph shown in Fig. 1.

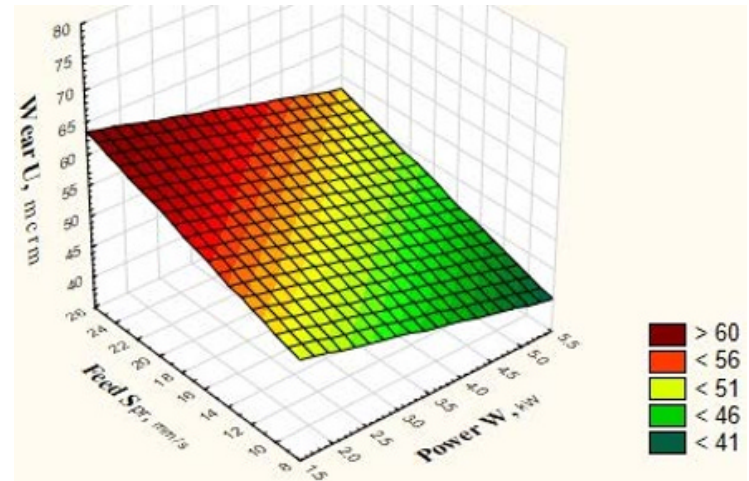

Fig. 1. Interactive effect of the radiation power and the longitudinal feed of the laser beam on the $P G-C P 4$ coating wear.

When assigning laser coating treatment modes, it is convenient to use $3 D-X Y Z$ contourgraphs. In relation to Fig. $13 D-X Y Z$ contour-graph is shown in Fig. 2.

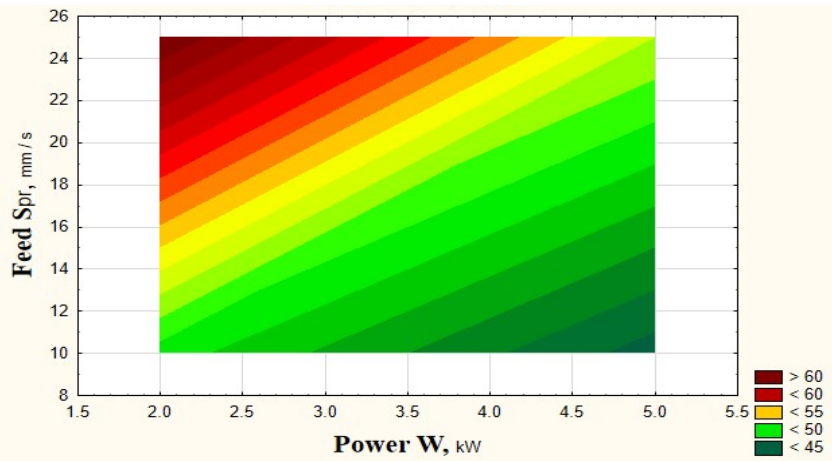

Fig. 2. $3 M-X Y Z$ contour-graph of the interactive effect of the radiation power and the longitudinal feed on the coating absolute wear.

The effect of the radiation power $W$ and the distance $L$ from the protective glass of the laser head to the treated surface of the sample on the absolute wear of the PG-CP4 coating is described by the formula:

$$
U=73.18-2.6(6) * W-0.16 * L
$$

The graphical interpretation of the regression equation (2) is represented by the $3 M-X Y Z$ surface-graph shown in Fig. 3. 


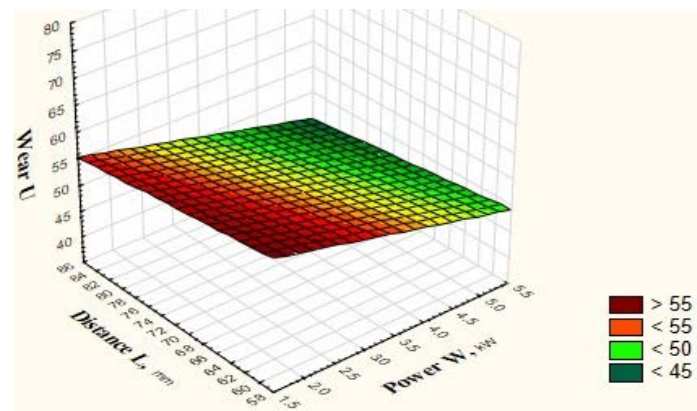

Fig. 3. Interactive effect of the radiation power and the distance from the protective glass of the laser head to the treated surface on the absolute wear of the $P G-C P 4$ coating.

The $3 D-X Y Z$ contour-graph of the influence of the radiation power and the distance from the protective glass of the laser head to the sample treated surface on the coating absolute wear has the form (Fig. 4).

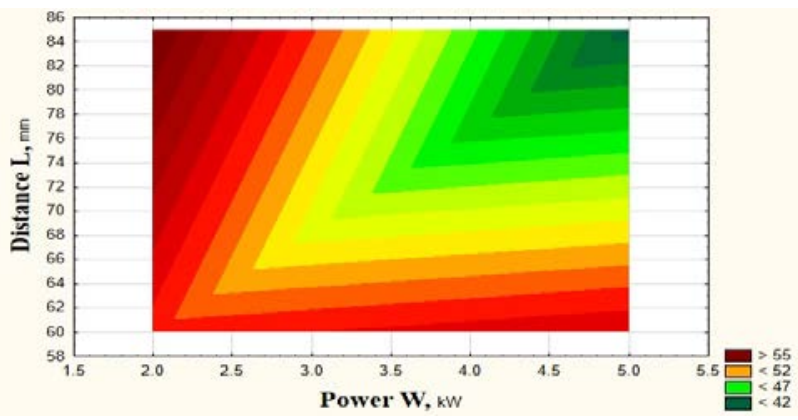

Fig. 4. 3D-XYZ contour-graph of the effect of the radiation power and the distance from the protective glass of the laser head to the treated surface on the coating absolute wear.

The effect of the laser beam Spr longitudinal feed and the distance $L$ from the protective glass of the laser head to the sample surface on the absolute wear of the PG-CP4 coating applied by plasma to the $H V G$ chrome steel and subjected to laser treatment is characterized by a multifactorial dependence of the form:

$$
U=51.6+0.7 * \operatorname{Spr}-0.16 * L
$$

A graphical interpretation of equation (3) is shown in Fig. 5.

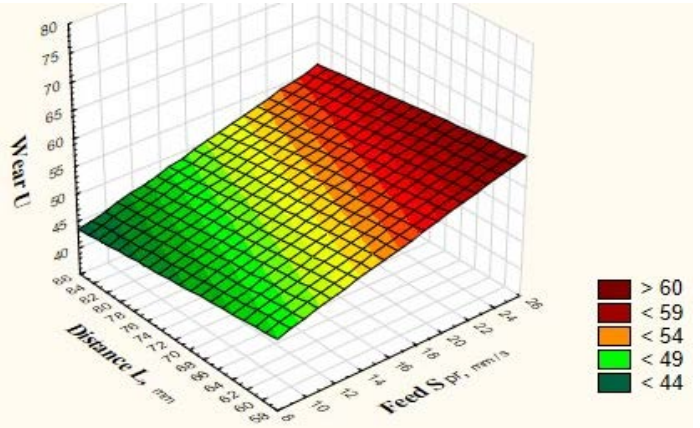

Fig. 5. $3 D-X Y Z$ surface-graph of the interactive effect of the longitudinal feed $S p r$ and the distance $L$ on the absolute wear of the $P G-C P 4$ coating. 
The $3 D-X Y Z$ contour-graph of the interactive effect of the radiation power and the distance from the protective glass of the laser head to the sample treated surface on the absolute wear of the coating has the form (Fig. 6).

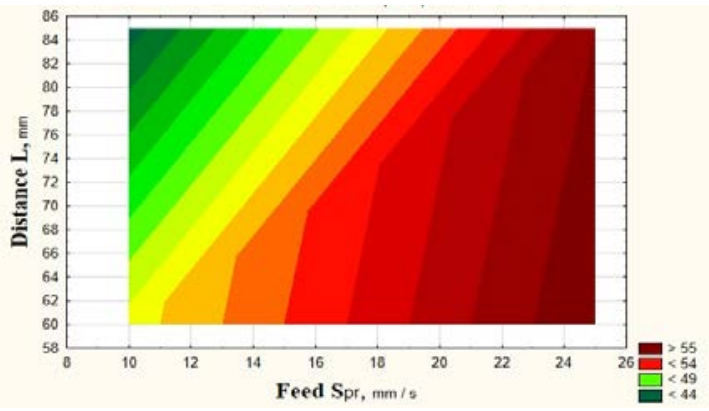

Fig. 6. $3 D-X Y Z$ contour-graph of the interactive effect of the longitudinal feed $\operatorname{Spr}$ and distance $L$ on the absolute wear of the $P G-C P 4$ coating.

The multi-factor model that relates the $P G-C P 4$ coating absolute wear to the independent factors of the laser processing process is represented by the regression equation in the code expression of the factors in the form:

$$
Y=51.0-4.0 * x_{1}+5.25 * x_{2}-2.0 * x_{3}
$$

When the independent factor $x_{1}$ increases and $x_{2}$ decreases the coating absolute wear applied to HVG steel decreases. The longitudinal feed has the greatest influence on the absolute wear, followed by the radiation power and the distance from the protective glass of the laser head to the treated surface. A regression equation was determined in which the absolute wear is associated with independent factors in their natural expression:

$$
U=59.7-2.6(6) * W+0.7 * S p r-0.16 * L
$$

The multifactorial dependence (5) is relevant for manufacturing enterprises implementing laser technologies, since the independent factors $W, S p r$ and $L$ in (5) have measurement units that are used by the personnel servicing the laser equipment. Taking into account the regression equation (5), an experiment was carried out with extended values of independent factors of the laser processing process, while data on the absolute wear of the PG-CP4 coating applied to HVG steel were obtained, presented in Table 2.

Table 2. Wear of the PG-CP4 coating applied to $H V G$ steel after laser treatment at extended values of input factors

\begin{tabular}{|c|c|c|c|}
\hline \multicolumn{3}{|c|}{ Factors } & Parameter \\
\hline$W, k W$ & $S p r, \mathrm{~mm} / \mathrm{s}$ & $L, \mathrm{~mm}$ & Wear $U, \mu \mathrm{m}$ \\
\hline 5 & 40 & 90 & 25 \\
\hline 5 & 20 & 55 & 51 \\
\hline
\end{tabular}

The wear of the $H V G$ steel surface layer in the state of delivery equals 50 micrometers. The wear of this steel with a $P G-C P 4$ coating applied by plasma and treated with laser radiation at $W=5 \mathrm{~kW}, S p r=25 \mathrm{~mm} / \mathrm{s}, L=85 \mathrm{~mm}$ was 40 micrometers. With the extended values of the input factors $W=5 \mathrm{~kW}, S p r=40 \mathrm{~mm} / \mathrm{s}, L=85 \mathrm{~mm}$, the absolute wear 
decreased to 25 micrometers (Table 2), which is twice less than the wear of steel in the delivery state. These data indicate a significant increase in the wear resistance of the $P G$ $C P 4$ coating applied by plasma to the HVG steel and subjected to laser treatment.

\section{Discussion}

It follows from formula (4) that the value of the $P G-C P 4$ coating absolute wear is most influenced by the longitudinal feed of the laser beam, with grow which the wear increases. Compared with the longitudinal feed of the laser beam, the effect of the radiation power on the absolute wear of the $P G-C P 4$ coating is less by $24 \%$, the effect of distance from the protective glass of the laser head to the treated surface of the sample is 4.4 times less. These data can be explained by the fact that with a decrease in the longitudinal feed and an increase in the laser radiation power, the temperature of the surface layer of the coating increases, its melting occurs, mixing of the chemical elements of the coating and the HVG steel. Due to the rapid heat removal deep into the sample, the surface layer is intensively cooled, as a result of which the latter get a higher hardness, which helps to reduce the absolute wear of the coating.

It follows from Table 1 that the laser treatment process of the PG-CP4 coating can proceed without (-) or with reflow (+) of the surface layer. In the reflow absence, local areas with pronounced small air gaps between the coating and the substrate were observed, which indicates their unsatisfactory adhesion. When reflowing, the such defect in the surface layer is not formed.

\section{Conclusion}

1. Based on the analysis of a priori information in the field of coating on products, it is established that traditional methods of improving the quality of coatings have exhausted themselves [3], and the known one-factor models cannot adequately describe multi-criteria and multi-parameter processes of real production.

2. The choice of independent factors of laser $H V G$ steel processing, which have a dominant influence on the optimization parameter (absolute wear of the coating), is justified, planning and implementation of a multifactorial experiment is carried out. As a result of experimental data statistical processing, a multifactorial model was established that relates the absolute wear $U$ of the $P G-C P 4$ coating to the radiation power $W$, the longitudinal feed Spr of the laser beam and the distance $L$ from the protective glass of the laser head to the sample treated surface.

3. The independent factor Spr has the greatest influence on the optimization parameter $U$, the factor $\mathrm{W}$ affects to a lesser extent (by 24)\%), the factor $\mathrm{L}$ has the least influence. The absolute wear of the PG-CP4 coating applied by plasma to the HVG steel and treated with laser radiation at $\mathrm{W}=5 \mathrm{~kW}, \mathrm{Spr}=40 \mathrm{~mm} / \mathrm{s}, \mathrm{L}=85 \mathrm{~mm}$, was 25 micrometers, which is two times less than the wear of this steel in the delivery state.

4. The use of the obtained scientific information allows us to quickly assign laser processing modes that provide a significant increase in the wear resistance of the $P G-C P 4$ functional coating.

The results of the research are recommended for use in enterprises that implement the processes of plasma and laser materials processing, as well as in design organizations that develop modern technological laser systems.

The study was conducted using the equipment of the Center for Collective Use (CCP) of promising and competitive technologies of the VlSU. 


\section{References}

[1] S.M. Nikiforova, M.S. Khadyev, A.S. Zhilin, et al, J. Basic research, 10 (1):(7377), (2016)

[2] O.N. Voitovich, I.O. Sokorov, Bul. of the Bel.-Rus. Un-ty, 2 (39):(6-14), (2013)

[3] Chirkov, Photonics Mag. 4:(28-31), (2008)

[4] A.T. Gabdrakhmanov, I.H. Israfilov, et al, IOP Conf. Ser.: Mat. Sci. and Eng. 240 (1):012015, (2015)

[5] V.A. Korotkov, M.V. Zlokazov, Bul. Friction and wear 35 (2):(52-55), (2014)

[6] V.A. Korotkov, M.V. Zlokazov, Bul. of Mech. Eng., (8):(59-61), (2014)

[7] M.V. Zlokazov, I.A. Alekseev, Mat. XXII Ural School of Met. Sci-Therm. Rus., Pub. house of OGTI:(201-203), (2014)

[8] V.A. Korotkov Plasma hardening of stamps, J. Forging and stamp, prod., (7):(4445), (2012)

[9] I.Yu. Sapronov, Dissertation, Moscow, MGTU (Stankin), (2007)

[10] V.G. Gusev Theory and practice of planning multi-factor experiments, Rus., VLSU (2010)

[11] V. Gusev, V. Morozov, D. Gavrilov, IOP Conf. Mat. Sci. and Eng., 896 (1):012119, (2020)

[12] V. Morozov, V. Gusev, IOP Conf. Mat. Sci. and Eng., 896 (1):012115, (2020)

[13] L.E. Afanasyeva, V.I. Yugov, Rus. J. Metallurgy (Metally), 13:(1293-1295), (2018)

[14] H. Shan-Ting, W. Hongliang, S.Gen, et al, Dep. of Mech. Eng., USA, Columbia Un-ty New York:10027, (2011)

[15] Korshunov L.G., Makarov A.V., Malygina I.Yu., et al, Rus. J. Phys. of met. and metallog. 103 (5):(536-548), (2007)

[16] V.Gorynin, C. Kondratev, V. Popov, Rus. J. Photonics 3:(26-32), (2010)

[17] Y.A. Kuznetsova, V.C. Laurynas, S.A.Guchenko, V.M. Yurov, Kazakhstan, Karaganda St. Un-ty J. Modern high-tech technol. 3:(39-42), (2015)

[18] Martn, J. Rodrguez, J.E. Fernández, et al, J. Wear 251 (1-12):(1017-1022), (2001)

[19] Almeida, F. Carvalho, P.A. Carvalho, et al, J. Surface \& Coatings Technology 200 (16-17):(4782-4790), (2006)

[20] A.V. Makarov, N.N. Soboleva, M.S. Gibzun, et al, AIP Conf. Proceed. 2053 (1):030037, (2018) 